C. Keuntje, P. Thomaser, W. A. Günthner

\title{
Ermittlung der Zykluszeit von Routenzügen
}

\author{
Zeitbausteinsystem auf Basis von MTM-Analysen und Probandenstudien
}

\begin{abstract}
In der Planung von Routenzugsystemen stellt die Zykluszeit eines Routenzugs eine wichtige Kenngröße dar. Sie ist erforderlich um die Anzahl der Routenzüge zu ermitteln, die für die Bewältigung der Transportaufgabe erforderlich sind. In diesem Beitrag wird hierzu ein Zeitbausteinsystem vorgestellt, das neben MTM-Analysen Ergebnisse aus umfangreichen Probandenstudien verwendet. Die exakte Berücksichtigung technischer und prozessualer Unterschiede ermöglicht eine belastbare Planung von Routenzugsystemen.
\end{abstract}

\section{Einleitung}

In einer steigenden Zahl von Unternehmen werden Routenzugsysteme zur Produktionsversorgung mit Großladungsträgern (GLT) und Kleinladungsträgern (KLT) eingesetzt. Durch Routenzüge, die in der Regel aus einem Zugfahrzeug und mehreren Anhängern bestehen, kann eine hochzyklische Produktionsversorgung mit kleinen Losgrößen realisiert werden. [1]

Im Rahmen der Planung von Routenzugsystemen kommt der Zykluszeit eine zentrale Bedeutung zu. Sie ist definiert als die Zeit, die ein Routenzug im Mittel für die Durchführung aller zugehörigen Tätigkeiten (z. B. Beladung und Materialbereitstellung) auf einer Tour benötigt und stellt den minimal möglichen Tourenstartabstand in einem Routenzugsystem dar. Auf Basis der Zykluszeit kann analog der VDI-Richtlinie 5586 in Abhängigkeit des zu erbringenden Systemdurchsatzes die Anzahl der erforderlichen Routenzüge ermittelt werden. [2]

Die Ermittlung der Zykluszeit eines Routenzugs wird insbesondere dadurch erschwert, dass eine Vielzahl von Routenzugtechniken und Prozessvarianten existieren, deren Einfluss auf die Zykluszeit vom Logistikplaner aufgrund unterschiedlicher Wechselwirkungen nicht in ausreichender Form berücksichtigt werden kann. Mit dem Ziel die Planungsphase methodisch zu unterstützen, wurde im 
Forschungsprojekt IntegRoute* eine Methodik zur Planung von Routenzugsystemen entwickelt. Als Teil der Planungsmethodik ermöglicht das nachfolgend vorgestellte Zeitbausteinsystem eine effiziente Ermittlung der Zykluszeit eines Routenzugsystems unter Berücksichtigung technischer und prozessualer Details.

\section{Einflussfaktoren auf die Zykluszeit von Routenzügen}

Basierend auf 26 Prozessaufnahmen von Routenzugsystemen wurden die eingesetzten Techniken analysiert und die entscheidenden Einflussfaktoren auf die Zykluszeit ermittelt. Die untersuchten Routenzugtechniken können basierend auf vergleichbaren Bereitstellvorgängen und für die Materialanstellung zusätzlich erforderlichen Elementen in den in Bild 1 dargestellten Kategorien zusammengefasst werden.

\begin{tabular}{|c|c|c|}
\hline Routenzug-Konzept & $\begin{array}{c}\text { Schematische Darstellung } \\
\text { (Spezifische technische Realisierung) }\end{array}$ & $\begin{array}{c}\text { Systemelement zur Anstellung der } \\
\text { Ladungsträger an den Senken }\end{array}$ \\
\hline Transportwagen & $\begin{array}{c}\text { Ein-/ Aufschubkonzept } \\
\text { Rollenverschiebe- }\end{array}$ & System
\end{tabular}

Bild 1: Übersicht über Routenzug-Konzepte (vgl. [2])

Während beim klassischen Transportwagen die Materialbereitstellung auf dem Transportwagen selbst geschieht, werden beim Ein-/ Aufschubkonzept zusätzliche Handschiebewagen verwendet. In Rollenverschiebe-Systemen werden GLT direkt auf fest an den Bereitstellorten installierte Rollenbahnen abgegeben.

Neben deutlichen Zeitunterschieden zwischen den drei vorgestellten RoutenzugKonzepten ergeben sich auch innerhalb der Konzepte deutliche Prozesszeitunterschiede. Diese sind in erster Linie auf verschiedene technische Ausführungen von Elementen wie Kupplung oder Hubmechanismus zurückzuführen. Als weitere Einflussfaktoren auf die Zykluszeit sind unterschiedliche Beladungsprozesse sowie Entfernungen und Spezifika des vom Routenzug zu 
befahrenden Layouts zu nennen. Auch die Anzahl und Anordnung der pro Sachnummer vorgehaltenen Ladungsträger und die ein- oder zweiseitige Materialbereitstellung haben einen deutlichen Einfluss auf den Zeitbedarf eines Routenzugs.

Im Rahmen von in Vorstudien durchgeführten Ergonomie-Untersuchungen wurde darüber hinaus deutlich, dass bei der Bereitstellung von GLT verschiedene technische Ausführungen des zu manipulierenden Elements zu abweichenden Handhabungszeiten führen können. Mit dem Ziel einer Quantifizierung dieser Beobachtung wurde eine Probandenstudie durchgeführt, deren Ergebnisse nachfolgend vorgestellt werden.

\section{Probandenstudie zum Ziehen und Schieben von Großladungsträgern}

In der mehrmonatigen Probandenstudie mit 42 Personen wurde das Ziehen und Schieben von GLT mit Gewichten zwischen 125 und 800 kg untersucht. Diese waren von den Probanden mit Wagen in einer Vielzahl von technischen Ausführungen auf einem festgelegten Parcours zu bewegen. Zu Details der Untersuchungsmethodik sei auf [3] verwiesen.

Durch systematische Variation der untersuchten Einflussparameter wurde die Stärke des Einflusses der einzelnen Parameter auf die Handhabungszeiten quantifiziert. Auf dieser Basis wurden Datenkarten für die Handhabungszeiten entwickelt, die ausschließlich die nachfolgend vorgestellten, signifikanten Parameter berücksichtigen.

Neben dem zu erwartenden deutlichen Einfluss des Gewichts hat auch das Material der Rollen am Wagen starke Auswirkungen auf die Zykluszeit. Unterschieden werden hier die Materialien Vollgummi, zwei Polyurethane unterschiedlicher Härte sowie Polyamid. Weiterhin konnte ein deutlicher Einfluss der Rollenanordnung gezeigt werden. Untersucht wurden jeweils vier Anordnungen für Ein-/ Aufschubkonzepte und Transportwagen, die sich aus der Kombination von drehbaren Lenkrollen und festen Bockrollen ergeben. Entgegen der etablierten Annahme konnte bei der Versuchsdurchführung auf fugenfreiem Industrieboden kein signifikanter Einfluss des Rollendurchmessers festgestellt werden.

Die Ergebnisse ermöglichen die exakte Bestimmung der Handhabungszeiten und stellen damit ein wichtiges Element für die Zeitermittlung eines Routenzugprozesses 
dar. Nachfolgend wird erläutert, in welcher Weise die Zeitwerte aus den Probandenstudien im entwickelten Zeitbausteinsystem berücksichtigt werden.

\section{Grundstruktur des Zeitbausteinsystems für Routenzüge}

Das entwickelte Zeitbausteinsystem basiert auf den Bausteinsystemen des Methods Time Measurement (MTM) „Universelles Analysiersystem“ (UAS, vgl. [4]) und den "Standardvorgängen Logistik" [5]. Zusätzlich wird das Zeitbausteinsystem durch die ermittelten Handhabungszeiten aus den vorgestellten Probandenstudien erweitert. Die Struktur des Bausteinsystems zur Ermittlung der Zykluszeit orientiert sich an der VDI-Richtlinie 5586 und beinhaltet die Abschnitte Beladung, Fahrt und Bereitstellung (vgl. [2]). Die Abschnitte beinhalten jeweils eine Vielzahl von Hauptbausteinen, wobei jeder Hauptbaustein eine Prozessvariante beschreibt. Die Zykluszeit eines Routenzugprozesses ergibt sich aus der Summe der für das Planungsszenario ausgewählten Hauptbausteine der drei Abschnitte.

In einem Hauptbaustein sind einzelne Tätigkeitsschritte sowie weitere untergeordnete Bausteine mit ihren jeweiligen Ausführungszeiten und -häufigkeiten enthalten (vgl. Bild 2). Um eine größere Bandbreite von Prozessvarianten abbilden zu können sind die Hauptbausteine dynamisch aufgebaut. Die Ausführungszeiten bzw. -häufigkeiten einzelner Tätigkeitsschritte sind daher in den Bausteinen nicht immer fix hinterlegt, sondern können entsprechend der Planungsaufgabe angepasst werden. Ebenso werden umfangreiche Tätigkeitsfolgen in untergeordneten Bausteinen modular in die Hauptbausteine eingebunden. 


\begin{tabular}{|c|c|c|c|c|c|}
\hline \multirow{2}{*}{$\frac{\text { BE-1 }}{\mathrm{Nr} .}$} & \multicolumn{5}{|c|}{ 1:1 THM-Behältertausch, Mehr-Behälter-Prinzip, nebeneinander } \\
\hline & Bezeichnung & Kode & TMU & $\mathrm{A} \times \mathrm{H}$ & $\Sigma$ TMU \\
\hline 1 & Feststellbremse des Schleppers ziehen & AZF & 120 & 0 & 0 \\
\hline 2 & vom Schlepper absteigen & KA & 25 & 1 & 25 \\
\hline 3 & zum Mittelpunkt des Anhängerverbands gehen & KA & 25 & 5 & 125 \\
\hline $4 a$ & 1:1 THM-Behältertausch, nebeneinander & $\underline{1: 1 . T H M . N}$ & 1906 & 1 & 1906 \\
\hline $4 \mathrm{~b}$ & 1:1 THM-Behältertausch, neben., mit Schiebehilfe & $\underline{1: 1 . T H M . N S}$ & 2800 & 0 & 0 \\
\hline 5 & zurück zum Schlepper gehen & KA & 25 & 5 & 125 \\
\hline 6 & auf Schlepper aufsteigen & KA & 25 & 1 & 25 \\
\hline 7 & Feststellbremse des Schleppers lösen & AZF & 120 & 0 & 0 \\
\hline & Tastendruck zum Start des Hubmechanismus & BA2 & 25 & 0 & 0 \\
\hline & Hubzeit am Anhänger abwarten & PT-HUB & 139 & 0 & 0 \\
\hline & Hubzeit auf dem Schlepper abwarten & PT-HUB & 139 & 1 & 139 \\
\hline & Seitenwand öffnen und schließen (Taxiwagen) & BB2 & 45 & 0 & 0 \\
\hline & vom Schleppersitz aufstehen und hinsetzen & AZB & 160 & 1 & 160 \\
\hline & & & & $\Sigma$ & 2505 \\
\hline
\end{tabular}

Bild 2: Aufbau eines Bausteins im Zeitbausteinsystem

Bild 2 zeigt einen Hauptbaustein für die Bereitstellung von GLT auf Transporthilfsmitteln im Mehr-Behälter-Prinzip nebeneinander. Die weiß hinterlegten Zellen beinhalten fixe Werte, während die grauen Zellen dynamisch an die Planungsparameter angepasst werden. Die Zeilen 4a und 4b beschreiben den eigentlichen Behältertausch durch den Routenzugfahrer. Der Zeitaufwand wird in einem untergeordneten Baustein berechnet und in den Hauptbaustein übernommen.

Neben dem exemplarisch vorgestellten Hauptbaustein sind in einer softwarebasierten Umsetzung des Zeitbausteinsystems alle erstellten Bausteine dynamisch miteinander verknüpft und ermöglichen trotz detaillierter Berücksichtigung technischer und prozessualer Unterschiede eine aufwandsarme Berechnung der Zykluszeit eines Routenzugs.

\section{Zeitbausteine für die Beladung des Routenzugs}

Im Bausteinsystem sind 20 unterschiedliche Beladungsprozesse abgebildet, wobei für manche Beladungsprozesse unterschiedliche technikabhängige Prozessvarianten möglich sind. Die unterschiedlichen Geh- und Fahrwege des Mitarbeiters werden im entsprechenden Hauptbaustein dynamisch an den Beladungsprozess angepasst. Dabei kann die Beladung eines Routenzugs grundsätzlich durch den Fahrer selbst, durch einen weiteren Mitarbeiter oder automatisiert erfolgen. 


\section{Fahrzeit des Routenzugs}

Die im Zeitbausteinsystem realisierte Logik zur Ermittlung der reinen Fahrtzeit des Routenzugs basiert auf einer Unterteilung der Fahrt in Anteile mit unterschiedlichen Geschwindigkeiten. Dieser Ansatz ermöglicht im Gegensatz zu pauschalen Zeitaufschlägen u. a. die Berücksichtigung der Tatsache, dass sich ein Routenzug mit fünf Anhängern länger in einer Kurve befindet als ein Routenzug mit nur zwei Anhängern. Da neben Kurven auch Rampen mit reduzierter Fahrgeschwindigkeit befahren werden und Zusatzaufgaben des Routenzugfahrers wie beispielsweise Sortiertätigkeiten existieren können, berechnet sich die Fahrzeit des Routenzugs auf Basis von (1).

$t_{\text {Fahrt }}=t_{v_{-} \text {Max }}+t_{v_{-} \text {Kurve }}+t_{v_{-} \text {Rampe }}+t_{\text {Zusatz }}$

Während bei der Kurven- und Rampengeschwindigkeit realtypische Vorgabewerte verwendet werden können, sind für die Ermittlung der Maximalgeschwindigkeit unterschiedliche Aspekte zu berücksichtigen. Neben Unternehmensvorgaben und zulässigen Höchstgeschwindigkeiten der Routenzuganhänger werden für die Zugmaschine typenspezifische Zuglastdiagramm verwendet, um die in Abhängigkeit der Anhängerlast realisierbare Maximalgeschwindigkeit zu bestimmen.

\section{Zeitbausteine für die Bereitstellung von Transportgut}

Die Auswahl eines geeigneten Bereitstellungskonzepts hängt eng mit der eingesetzten Anhängertechnik zusammen. Grundsätzlich lassen sich die Bereitstellungskonzepte in fünf Oberkategorien unterteilen:

- GLT auf Transporthilfsmittel im Ein-/ Aufschubkonzept

- GLT auf Transportwagen

- GLT mittels Rollenverschiebesystem

- Einzelne KLT

- Einzelne behälterlose Transportgüter

Im Zeitbausteinsystem sind 16 unterschiedliche Bereitstellprozesse abgebildet, wobei für einige Bereitstellprozesse diverse technikabhängige Prozessvarianten möglich sind.

\section{Einfluss des Gesamtgewichts}


Im Zeitbausteinsystem werden die Zeitaufwände für das Ziehen und Schieben von Transportwagen/ Handschiebewagen aus den Ergebnissen der durchgeführten Probandenstudie verwendet. Die Entwicklung der Handhabungszeit in Abhängigkeit des zu manipulierenden Gesamtgewichts ist in Bild 3 für die Bereitstellung im MehrBehälter-Prinzip hintereinander dargestellt.

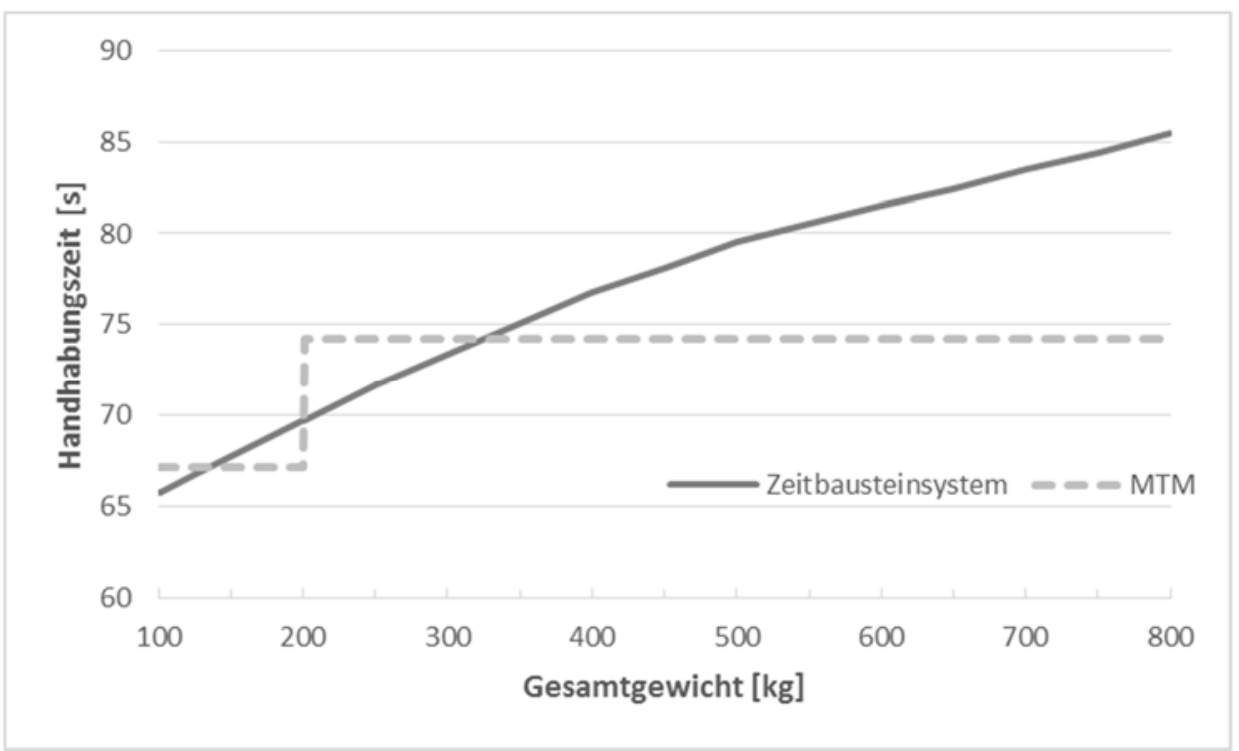

Bild 3: Einfluss des Gesamtgewichts auf die Handhabungszeiten

Während die Handhabungszeit im Zeitbausteinsystem mit zunehmendem Gewicht stetig ansteigt, bleibt sie bei Verwendung der MTM-Datenkarte „Transportwagen“ ab einem Gewicht von 200 kg konstant. Die Gegenüberstellung macht deutlich, dass insbesondere in höheren Gewichtsbereichen die Verwendung des entwickelten Zeitbausteinsystems zu einer deutlich genaueren Zeitermittlung führt.

\section{Gegenüberstellung verschiedener Routenzugkonzepte}

Im folgenden Planungsszenario werden die in Bild 1 vorgestellten Routenzugkonzepte auf Basis derselben Ausgangsituation miteinander verglichen. Betrachtet wird ein System zur Bereitstellung von GLT mit einem aus fünf Anhängern bestehenden Routenzug. Das Gewicht eines Vollgutwagens beträgt 580 kg, ein Leergutwagen wiegt $200 \mathrm{~kg}$. Für alle verbleibenden Detailparameter werden praxistypische Werte angenommen. Die mit dem Zeitbausteinsystem berechneten Zykluszeiten sind in Bild 4 dargestellt. 


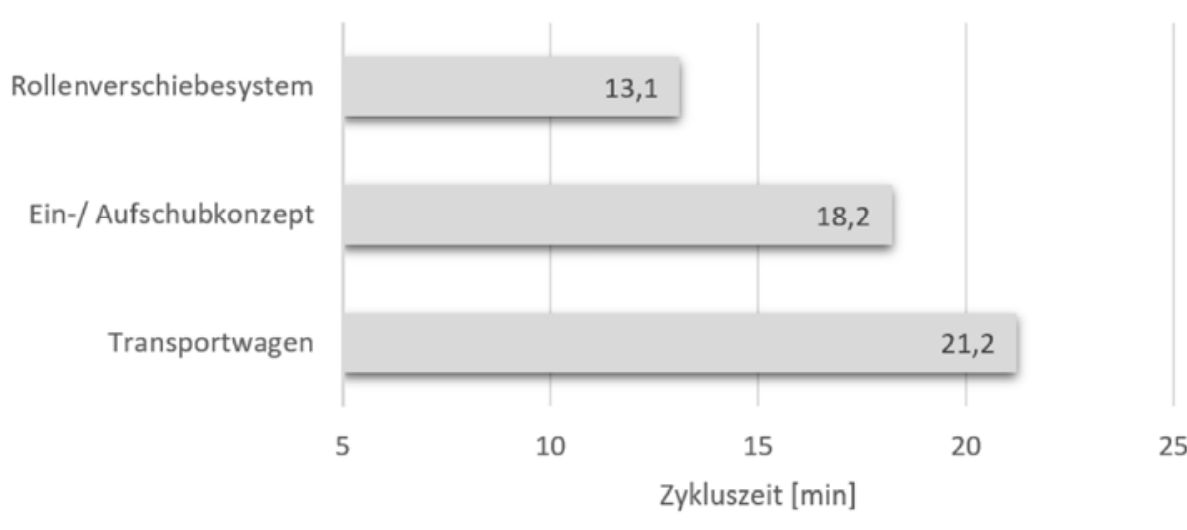

Bild 4: Vergleich der Zykluszeiten mit unterschiedlichen Anhängerkonzepten

Es zeigt sich deutlich, dass die Abgabe von GLT auf fest an den Senken installierten Rollenbahnen zu einer deutlich kürzeren Zykluszeit des Rollenverschiebesystems im Vergleich zum Ein-/ Aufschubkonzept führt. Die Vielzahl der bei Transportwagen erforderlichen Kuppelvorgänge führen zu den im Vergleich höchsten Zykluszeiten.

\section{Einfluss der Rollenkonfiguration}

Das Zeitbausteinsystem ermöglicht unter anderem die Berücksichtigung der Auswirkungen unterschiedlicher Rollenkonfigurationen der Transportwagen/ Handschiebewagen auf die Zykluszeit eines Routenzugs. Das folgende Beispiel verdeutlicht den signifikanten Einfluss der Rollenkonfiguration auf die Handhabungszeiten eines Handschiebewagens. Betrachtet wird erneut die Bereitstellung eines GLT im Mehr-Behälter-Prinzip hintereinander. Als Rollenanordnung werden vier Lenkrollen und zwei Lenkrollen mit zwei Bockrollen miteinander verglichen. Bezüglich des Rollenmaterials wird sowohl Polyamid als auch Vollgummi verwendet. Als Referenz findet sich die mittels einer klassischen MTM-Analyse ermittelte Handhabungszeit für den Bereitstellprozess, die für alle betrachteten Varianten identische Zeitwerte liefert, ebenfalls in Bild 5. 


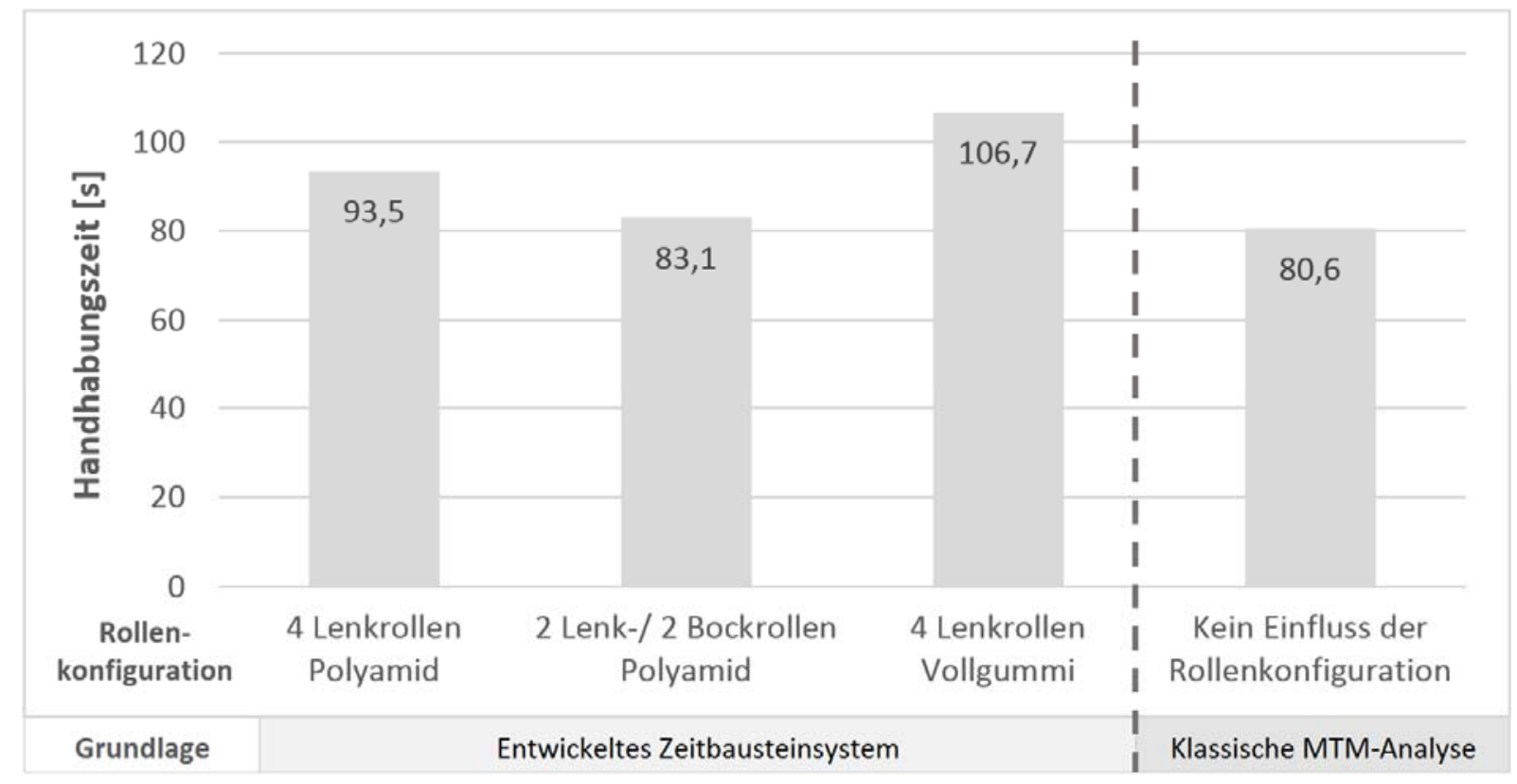

Bild 5: Einfluss der Rollenkonfiguration auf die Handhabungszeit

Für die Bereitstellung eines GLT beträgt der Zeitunterschied zwischen der günstigsten und ungünstigsten Rollenkonfiguration 23 Sekunden. Im Vergleich zum Ergebnis einer klassischen MTM-Analyse beträgt der maximale Zeitunterschied 26 Sekunden. In einem großen Routenzugsystem mit einem Tagesbedarf von z. B. 3000 GLT kann sich dadurch allein beim Zeitanteil der Bereitstellung an den Senken ein Unterschied von mehr als 19 Stunden Arbeitszeit ergeben. Dies macht den Einfluss von technischen Detailentscheidungen auf die Zykluszeit und damit Wirtschaftlichkeit eines Routenzugsystems deutlich und unterstreicht den Mehrwert des vorgestellten Zeitbausteinsystems.

\section{Zusammenfassung und Ausblick}

Das entwickelte Zeitbausteinsystem ermöglicht durch die softwarebasierte Umsetzung trotz einer detaillierten Abbildung von technischen und prozessualen Unterschieden eine aufwandsarme Berechnung der Zykluszeit von Routenzügen. Somit können in der Planung verschiedene Systemvarianten detailliert gegenübergestellt und Effekte transparent gemacht werden, die derzeit insbesondere in frühen Planungsphasen nicht abgebildet werden können.

Die auf Basis der durchgeführten Probandenstudien mögliche Berücksichtigung des Einflusses technischer Details wie des Rollenmaterials oder der Rollenanordnung des zu manipulierenden Objekts befähigt Logistikplaner in Planungsprojekten deutlich fundiertere Auswahlentscheidungen zu treffen. 
Im Rahmen des Forschungsprojekts IntegRoute wurde das vorgestellte Zeitbausteinsystem in eine ganzheitliche Planungsmethodik integriert und schafft somit die Grundlage für eine belastbare Dimensionierung und Projektkostenrechnung. Neben der Betrachtung der Wirtschaftlichkeit werden mit dem Ziel einer ganzheitlichen Bewertung von Planungsvarianten ebenfalls eine Vielzahl von nicht monetär quantifizierbaren Kriterien wie Ergonomie, Flexibilität oder Robustheit betrachtet.

Die entwickelte Planungsmethodik ermöglicht schließlich eine durchgehende methodische Unterstützung von Logistikplanern bei der Gestaltung von Routenzugsystemen, wobei auch Detailfragen bereits in frühen Planungsphasen mit höherer Sicherheit berücksichtigt werden können.

\section{Literatur}

1. Günthner, W. A.; Galka, S.; Klenk, E.; Knössl, T.; Dewitz, M.: Stand und Entwicklung von Routenzugsystemen für den innerbetrieblichen Materialtransport. Lehrstuhl für Fördertechnik Materialfluss Logistik, Garching 2012.

2. Verein Deutscher Ingenieure: Routenzugsysteme - Grundlagen, Gestaltung und Praxisbeispiele. VDI-Richtlinie Nr. 5586, 2016.

3. Keuntje, C.; Heiß, J.; Gangkofner, P.; Günthner, W. A.: Experimentelle Untersuchungen zur Ergonomie von Handschiebewagen in Routenzugsystemen. In: 11. Hamburger Staplertagung. Hrsg.: R. Bruns. Hamburg 2016.

4. Deutsche MTM-Vereinigung e.V.: MTM-UAS. Deutsche MTM-Vereinigung, Hamburg, 2004.

5. Deutsche MTM-Vereinigung e.V.: Handbuch MTM-Logistik. Deutsche MTMVereinigung, Hamburg, 2007.

\section{*) Förderhinweis}

Dieser Beitrag entstand im Rahmen des IGF-Forschungsvorhabens IntegRoute (Fördernummer 18136 N). Das Forschungsvorhaben der Bundesvereinigung Logistik (BVL) e.V. wurde über die AiF im Rahmen des Programms zur Förderung der industriellen Gemeinschaftsforschung und -entwicklung (IGF) vom Bundesministerium für Wirtschaft und Technologie aufgrund eines Beschlusses des Deutschen Bundestages gefördert. 


\section{Die Autoren dieses Beitrags}

M. Sc. Christopher Keuntje, geb. 1982, studierte Maschinenbau und Management an der Fakultät für Maschinenwesen der TU München. Seit Abschluss seines Studiums ist er als wissenschaftlicher Mitarbeiter am Lehrstuhl für Fördertechnik Materialfluss Logistik (fml) der TU München beschäftigt. Im Rahmen seiner Forschungstätigkeit auf dem Gebiet der Routenzugtechnik hat er als Co-Autor an der Erarbeitung der VDI-Richtlinie 5586 - Routenzugsysteme mitgewirkt.

M.Sc. Patrick Thomaser, geb. 1988, studierte Produktion und Logistik an der Freien Universität Bozen sowie an der Fakultät für Maschinenwesen der TU München. Seit dem Abschluss seines Studiums arbeitet er im Werksleitungsteam bei der Dr. Schär AG in Burgstall (IT).

Prof. Dr.-Ing. Dipl.-Wi.-Ing. Willibald A. Günthner leitet seit 1994 den Lehrstuhl für Fördertechnik Materialfluss Logistik (fml) an der TU München. Er ist Gründungsmitglied der Wissenschaftlichen Gesellschaft für Technische Logistik e.V., stellvertretender Vorsitzender des wissenschaftlichen Beirats der BVL.

\section{Summary}

Calculation of the cycle time of tugger trains - time module system based on MTM analyses and experimental studies

In the planning of tugger train systems the cycle time presents an important parameter. It is required to determine the number of tugger trains needed to meet the given transport task. In this article proposes a time module system that bases on methods time measurement and findings of experimental studies. Taking the technical and procedural differences into account the system allows for a reliable planning of tugger train systems.

\section{Bildunterschriften}

Bild 1: Übersicht über Routenzug-Konzepte (vgl. [2])

Figure 1: Overview of tugger train concepts (c.f. [2])

Bild 2: Aufbau eines Bausteins im Zeitbausteinsystem

Figure 2: Structure of a building block in the time module system

Bild 3: Einfluss des Gesamtgewichts auf die Handhabungszeiten 
Figure 3: Influence of the weight on the handling times

Bild 4: Vergleich der Zykluszeiten mit unterschiedlichen Anhängerkonzepten

Figure 4: Comparison of cycle times with varying tugger train concepts

Bild 5: Einfluss der Rollenkonfiguration auf die Handhabungszeit

Figure 5: Impact of the configuration of the castors on the handling time 